\title{
Coincidence Summing Correction for Cylinder and Marinelli Beaker Sources by Monte Carlo Simulation
}

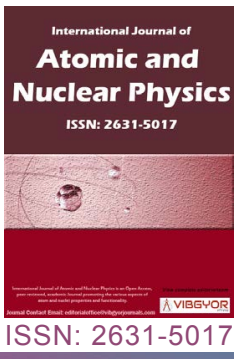

Waseem Khan, Chaohui He* and Yu Cao

Department of Nuclear Science and Technology, Xi'an Jiaotong University, China

\begin{abstract}
Coincidence summing effects arises when two or more $y$-rays are emitted in a cascade from an excited nucleus and are detected within the resolving time of the detector. Without correction of such effects, the activity of radionuclides cannot be accurately determined. For the correction of summing effects, a new simulation method in GEANT4 was established to simulate the coincidence summing correction factors $\left(\mathrm{CSF}_{\text {simu }}\right)$ for an HPGe detector. In the simulation, a cylindrical and Marinelli beaker source containing several radionuclides were used with different volumes, covering the energy range from $59.50 \mathrm{keV}$ to $1836.01 \mathrm{keV}$. In the case of volumetric sources, the coincidence summing correction factors for two nuclides $\left({ }^{60} \mathrm{Co}\right.$ and ${ }^{88} \mathrm{Y}$ ) were calculated from the efficiencies at different points throughout the source volume. The dependence of the coincidence correction factor on the sample density was also carried out for some particular nuclide and photon energy. The same methodology of coincidence summing correction factor was applied for the complex decay scheme of ${ }^{133} \mathrm{Ba}$ and ${ }^{152} \mathrm{Eu}$ obtained a good agreement with the experimental results.
\end{abstract}

Keywords

GEANT4, HPGe detector, Coincidence summing, Marinelli beaker sources

\section{Introduction}

Y-ray spectrometry with HPGe detector is widely used to determine the activity of radionuclides in environmental samples. The accurate assessment of the activity of radionuclides would require a minimum source-detector distance to reduce the detection limit of the measuring system. The coincidence summing effect is more significant at a small source-detector distance because the probability of two $\gamma$-rays reaching the detector at the same time cannot be negligible at such distance.
The coincidence summing effect changes in the count from the peaks corresponding to the two $\gamma$-rays and nuclides activity become inaccurate if no correction is performed. For the correction of such effects, the contribution of total efficiency is also required with the full energy peak efficiency. Various groups used different calibration techniques and obtained the coincidence summing correction factors (CSFs) from the total efficiency. Debertin \& Schötzig [1] used the experimental technique and calculated the CSF from the total efficiency (the ratio of the total number of pulses recorded to the

*Corresponding author: Chaohui He, Department of Nuclear Science and Technology, School of Energy and Power Engineering, Xi'an Jiaotong University, Xi'an 710049, China

Accepted: May 18, 2019; Published: May 20, 2019

Copyright: (C) 2019 Khan W, et al. This is an open-access article distributed under the terms of the Creative Commons Attribution License, which permits unrestricted use, distribution, and reproduction in any medium, provided the original author and source are credited.

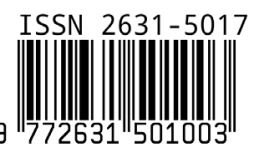

Khan et al. Int J At Nucl Phys 2019, 4:011

Citation: Khan W, He C, Cao Y (2019) Coincidence Summing Correction for Cylinder and Marinelli Beaker Sources by Monte Carlo Simulation. Int J At Nucl Phys 4:011 
number of photons emitted by the source). Practically, the total efficiency curve is difficult to achieve due to the single $\gamma$-ray emitting nuclides and preparation of standard sources. Several authors used the analytical approaches for the calculation of the CSF from the total efficiency [2-9]. These approaches required information about the nuclear decay parameters such as the mode of parent nuclide decay, conversion factors, and the probability for the $\psi$-ray transition from one energy level to another etc. Z Wang, et al. [10] used the Monte Carlo code MCNP and simulated the total efficiencies for the correction of coincidence summing effect. They used point source to test the coincidence summing correction method and observed a coincidence peak efficiency of at small source-detector distances. However such analysis is difficult to achieve for the close geometry measurements and large volume samples because in volumetric sources the contribution of the scattered $\nu$-rays to the total efficiency cannot be neglected [11]. Many authors proposed an approach of point sources positioned in the matrix of the extended source for the calculation of peak, total efficiencies and CSF [4,1215]. Tk Wang, et al. [4] include the effects of volume factor in the CSF values and observed a good agreement between calculated and experimental results. Recent techniques $[16,17]$ in GEANT4 were good for the calculation of CSF, but such computational techniques required elaborate work in its implementation.
The aim of this paper is to develop a simplest and modest method in Geant4 for the coincidence summing correction factors $\left(\mathrm{CSF}_{\text {simu }}\right.$ ) of the extended sources. The $\mathrm{CSF}_{\text {simu }}$ values were compared with the calculated and experimental results reported by Wang, et al. [4] and obtained good agreements.

\section{Materials and Methods}

GEANT4 [18] toolkit includes simulation of the electromagnetic interaction of charged particle, gamma, and optical photons. The code follows the history of each individual primary photon until its energy dissipated in the detector and produces secondary particles as a result of photoelectric effect, Compton effect, pair production interaction, multiple scattering, bremsstrahlung, and ionization. The secondary electrons formed by photon interaction processes were also taken into consideration in the simulation. GEANT4 electromagnetic physics class was used in the simulation since the energy limit for the electromagnetic process is $10 \mathrm{keV}$ to $100 \mathrm{TeV}$. Therefore, Ge X-rays of energy below 10 $\mathrm{keV}$ cannot be processed. GEANT4 also includes low-level electromagnetic processes that can simulate a particle down to $250 \mathrm{eV}$. The number of total histories ( $10^{7}$ primary photons) was considered for the simulation to obtain a statistical uncertainty of no more than $0.1 \%$. All the photon energies emitted by the source were individually simulated for the source-detector geometries.

Only the $\mathrm{\gamma}$-rays, which deposit their full energy

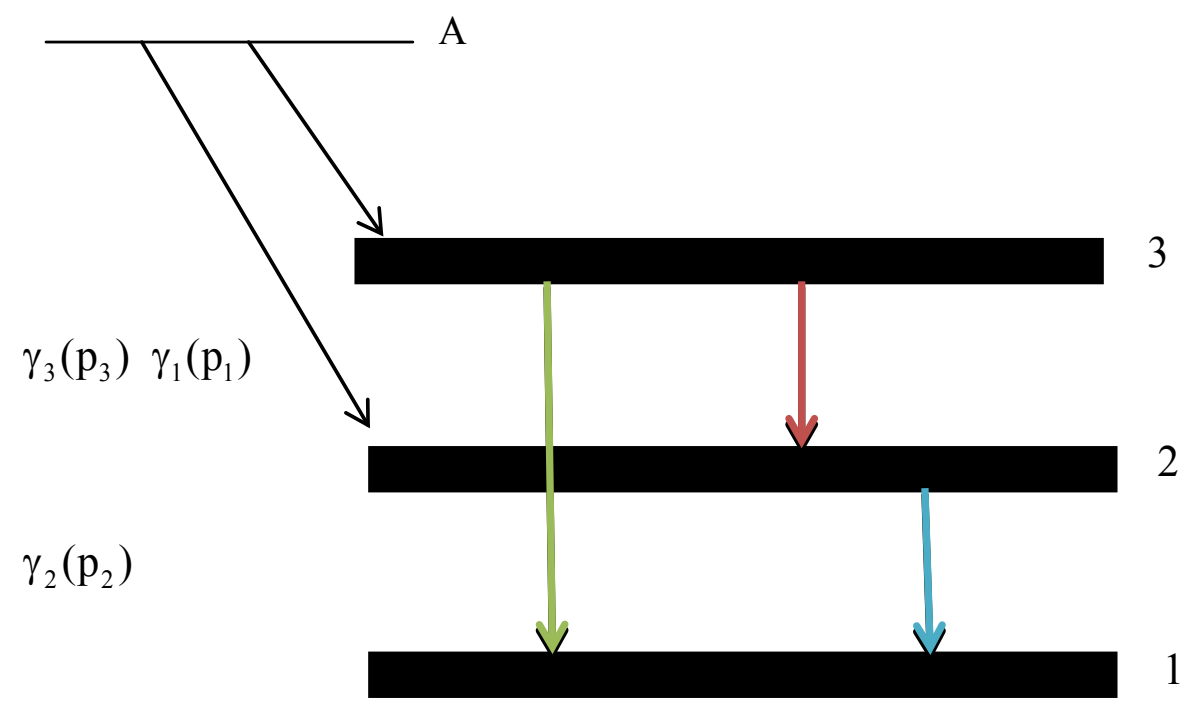

B

Figure 1: A typical decay scheme to show the coincidence summing effect. 
in the active volume of the detector, were considered for the evaluation of full energy peak efficiency. The simulated full energy peak efficiencies are obtained from

$$
\varepsilon=\frac{Q}{M}
$$

where $\varepsilon$ is the full energy peak efficiency, $Q$ is the number of counts that deposit their full energy in the active detector volume, and $\mathrm{M}$ is the number of total simulated $\boldsymbol{\gamma}$-rays counts for a given energy, E.

In order to simulate the total efficiencies and CSFs, a detailed decay scheme is considered as shown in Figure 1 . The nuclide $A$ decays to the two excited states of $B$. The two excited states deexcite by the emission of three $\gamma$-rays $\gamma_{1}(3 \rightarrow 2), \gamma_{2}(2 \rightarrow 1), \gamma_{3}(3 \rightarrow 1)$ with their respective probabilities as $\mathrm{P}_{1}, \mathrm{P}_{2}$, and $\mathrm{P}_{3}$.

In absence of coincidence summing, the count rate is given by;

$$
\mathrm{N}_{1}=\mathrm{A} \mathrm{p}_{1} \varepsilon_{1}
$$

Where $A$ is the source activity, $p_{1}$ is the emission probability with energy $E_{1}$ and $\varepsilon_{1}$ is peak efficiency for $v_{1}$ with $\mathrm{E}_{1}$.

The count rate $N_{1}^{*}$ in the recorded full energy peak will be smaller than $\mathrm{N}_{1}$. So the in presence of coincidence summing the count rate is given by

$$
\mathrm{N}_{1}^{*}=\mathrm{A} \mathrm{p}_{1} \varepsilon_{1}-\mathrm{A} \mathrm{p}_{1} \varepsilon_{1} \varepsilon_{\mathrm{T} 2}
$$

Where $\varepsilon_{\mathrm{T} 2}$ is the total detection efficiency for $\gamma_{2}$. The $\mathrm{CSF}_{\text {Simu }}$ for $\gamma_{1}$ is given by

$$
\begin{aligned}
& \frac{\mathrm{N}_{1}}{\mathrm{~N}_{1}^{*}}=\frac{1}{1-\varepsilon_{\mathrm{T} 2}} \\
& \frac{\mathrm{N}_{1}^{*}}{\mathrm{~N}_{1}}=1-\varepsilon_{\mathrm{T} 2} \\
& \mathrm{or}^{\mathrm{a}} \\
& \mathrm{CSF}_{\text {Simu }}^{\mathrm{a}}=1-\varepsilon_{\text {Tsimu }}^{\mathrm{b}}
\end{aligned}
$$

Similarly for $\mathrm{Y}_{2}$

$$
\begin{aligned}
& \mathrm{N}_{2}=\mathrm{App}_{2} \varepsilon_{2} \\
& \mathrm{~N}_{2}^{*}=\mathrm{Ap}_{2} \varepsilon_{2}-\mathrm{A} \mathrm{p}_{1} \varepsilon_{2} \varepsilon_{\mathrm{T} 1} \\
& \frac{\mathrm{N}_{2}}{\mathrm{~N}_{2}^{*}}=\frac{1}{1-\frac{\mathrm{p}_{1}}{\mathrm{p}_{2}} \varepsilon_{\mathrm{T} 1}} \\
& \frac{\mathrm{N}_{2}^{*}}{\mathrm{~N}_{2}}=1-\frac{\mathrm{p}_{1}}{\mathrm{p}_{2}} \varepsilon_{\mathrm{T} 1}
\end{aligned}
$$

$$
\begin{aligned}
& \text { Or } \\
& \mathrm{CSF}_{\text {Simu }}^{\mathrm{b}}=1-\frac{\mathrm{p}_{1}}{\mathrm{p}_{2}} \varepsilon_{\text {Tsimu }}^{\mathrm{a}}
\end{aligned}
$$

Where $\mathrm{CSF}_{\text {Simu }}^{\mathrm{a}}$ and $\mathrm{CSF}_{\text {Simu }}^{\mathrm{b}}$ are the simulated coincidence summing correction factors, $\varepsilon_{\text {Tsimu }}^{\mathrm{a}}$ and $\varepsilon_{\text {Tsimu }}^{\mathrm{b}}$ are the simulated total efficiencies of $1173.24 \mathrm{keV}$ (a) and $1332.50 \mathrm{keV}$ (b) respectively, similarly for ${ }^{88} \mathrm{Y},{ }^{133} \mathrm{BA}$ and ${ }^{152} \mathrm{Eu}$.

The coincidence summing effects become more complicated for the extended volume sources. In this case, the correction factor not only depends on the peak and total efficiencies but also on the source volume and the differential efficiency distributions within the source. For volume sources, the $\mathrm{CSF}_{\text {Simu }}$ is given by

$$
\mathrm{CSF}_{\text {Simu }}^{\mathrm{a}}=\int \rho \varepsilon_{1}\left(1-\varepsilon_{\text {Tsimu }}^{\mathrm{b}}\right) \mathrm{d} \rho / \int \rho \varepsilon_{1} \mathrm{~d} \rho
$$

$$
\mathrm{CSF}_{\text {Simu }}^{\mathrm{b}}=\int \rho \varepsilon_{2}\left(1-\frac{\mathrm{p}_{1}}{\mathrm{p}_{2}} \varepsilon_{\text {Tsimu }}^{\mathrm{a}}\right) \mathrm{d} \rho / \int \rho \varepsilon_{2} \mathrm{~d} \rho
$$

Or, as a summation,

$$
\begin{gathered}
\mathrm{CSF}_{\text {Simu }}^{\mathrm{a}}=1-\left[\sum \rho_{\mathrm{i}} \varepsilon_{1} \varepsilon_{\text {Tsimu }}^{\mathrm{b}} \mathrm{d} \rho / \sum \rho_{\mathrm{i}} \varepsilon_{1} \mathrm{~d} \rho\right] \\
\mathrm{CSF}_{\text {Simu }}^{\mathrm{b}}=1-\left[\sum \rho_{\mathrm{i}} \varepsilon_{2} \frac{\mathrm{p}_{1}}{\mathrm{p}_{2}} \varepsilon_{\text {Tsimu }}^{\mathrm{a}} \mathrm{d} \rho / \sum \rho_{\mathrm{i}} \varepsilon_{2} \mathrm{~d} \rho\right]
\end{gathered}
$$

Where $\rho_{\mathrm{i}}$ are the radial positions of the point sources from the beaker axis. Eq 14 and Eq 15 can be written as

$$
\begin{aligned}
& \mathrm{CSF}_{\text {Simu }}^{\mathrm{a}}=1-\left\langle\mathrm{J}_{1}\right\rangle \\
& \text { For } \mathrm{h}_{1,} \\
& \mathrm{~J}_{1 \mathrm{~h}_{1}}=\sum \rho_{\mathrm{i}} \varepsilon_{1} \varepsilon_{\text {Tsimu }}^{\mathrm{b}} \mathrm{d} \rho / \sum \rho_{\mathrm{i}} \varepsilon_{1} \mathrm{~d} \rho
\end{aligned}
$$

For the whole volume source height,

$$
\left\langle\mathrm{J}_{1}\right\rangle=\frac{\sum_{\mathrm{i}=1}^{3} \mathrm{~J}_{1 \mathrm{~h}_{\mathrm{i}}}}{3}
$$

Where $h_{i}$ are the different distances from the beaker bottom. Similarly,

$$
\begin{aligned}
& \mathrm{CSF}_{\text {Simu }}^{\mathrm{b}}=1-\left\langle\mathrm{J}_{2}\right\rangle \\
& \mathrm{J}_{2 \mathrm{~h}_{1}}=\sum \rho_{\mathrm{i}} \varepsilon_{2} \varepsilon_{\text {Tsimu }}^{\mathrm{a}} \mathrm{d} \rho / \sum \rho_{\mathrm{i}} \varepsilon_{2} \mathrm{~d} \rho
\end{aligned}
$$




$$
\left\langle\mathrm{J}_{2}\right\rangle=\frac{\sum_{\mathrm{i}=1}^{3} \mathrm{~J}_{2 \mathrm{~h}_{\mathrm{i}}}}{3}
$$

Where $\left\langle\mathrm{J}_{1}\right\rangle$ and $\left\langle\mathrm{J}_{2}\right\rangle$ are the average of 15-point integration of efficiencies.

To calculate the coincidence summing correction for all volumes, first, the cylinder and Marinelli beaker volumes are divided into three volumes $\left(h_{1}\right.$, $h_{2}$, and $h_{3}$ ) and then further subdivided into 5 volume elements $J_{1 h_{1}}$ for each $\left(h_{1}, h_{2}\right.$, and $\left.h_{3}\right)$. Every single nuclide in ${ }^{60} \mathrm{Co},{ }^{88} \mathrm{Y}$, and ${ }^{152} \mathrm{Eu}$ considered as a point source with their respective photon energies and placed at 15 positions within the source volume with three different distances $\left(h_{1}, h_{2}\right.$, and $h_{3}$ ) from the beaker bottom. To get ${ }_{1 \mathrm{~h}}$ for 898.02 $\mathrm{keV}$ or $1173.24 \mathrm{keV}$ at volume source height $h_{1^{\prime}}$ first computed the $\varepsilon$ and $\varepsilon_{\text {Tsimu }}^{\mathrm{b}}$ (at $1836.01 \mathrm{keV}$ or $1332.50 \mathrm{keV}$ ) values at 5 different positions in the source volume and then computed the 5-point integration (i.e., multiplied each value by $\rho_{i}$, summed them, and divided by the sum of the $\rho_{i} \varepsilon$ ). Similarly, calculated $J_{1 h_{2}}$ (5-point integration of efficien- cies) and $J_{1 h_{3}}$ (5-point integration of efficiencies) at height $h_{2}$ and $h_{3}$ respectively and averaged them to get $\left\langle\mathrm{J}_{1}\right\rangle$ at 15 volume elements except for the axial position of the beaker. The $\varepsilon$ and $\varepsilon_{\text {Tsimu }}$ value does not change with the further subdivision of the beaker volume. The same method was applied for $1836.01 \mathrm{keV}$ and $1332.50 \mathrm{keV}$ to obtain $\left\langle\mathrm{J}_{2}\right\rangle$ but used $\varepsilon_{\text {Tsimu }}^{\mathrm{a}}(898.02 \mathrm{keV}$ and $1173.24 \mathrm{keV})$ respectively in this case. The CSF values were also obtained for ${ }^{133} \mathrm{Ba}(276.39 \mathrm{keV}, 302.85 \mathrm{keV})$ and ${ }^{152} \mathrm{Eu}$ (778.9 KeV, $964.0 \mathrm{keV}$ and $444.0 \mathrm{KeV}$ ) nuclides using the same procedures.

The detector considered for $\mathrm{MC}$ simulation was a p-type coaxial HPGe detector (Canberra). The main parameters of the detector provided by the manufacturer are shown in Figure 2. No information was available by the manufacturer about whether the Ge crystals had rounded edges. Sharp edges of the crystals were assumed in the simulation. First, a cylindrical beaker source of diameter $(D=43.4 \mathrm{~mm}$ ) filled with gamma radionuclides aqueous solution of volumes V1 $(50 \mathrm{~mL}), \mathrm{V} 2(100 \mathrm{~mL}), \mathrm{V} 3(200 \mathrm{~mL})$, and V4 $(300 \mathrm{~mL})$ was used to obtain the values. A Marinelli

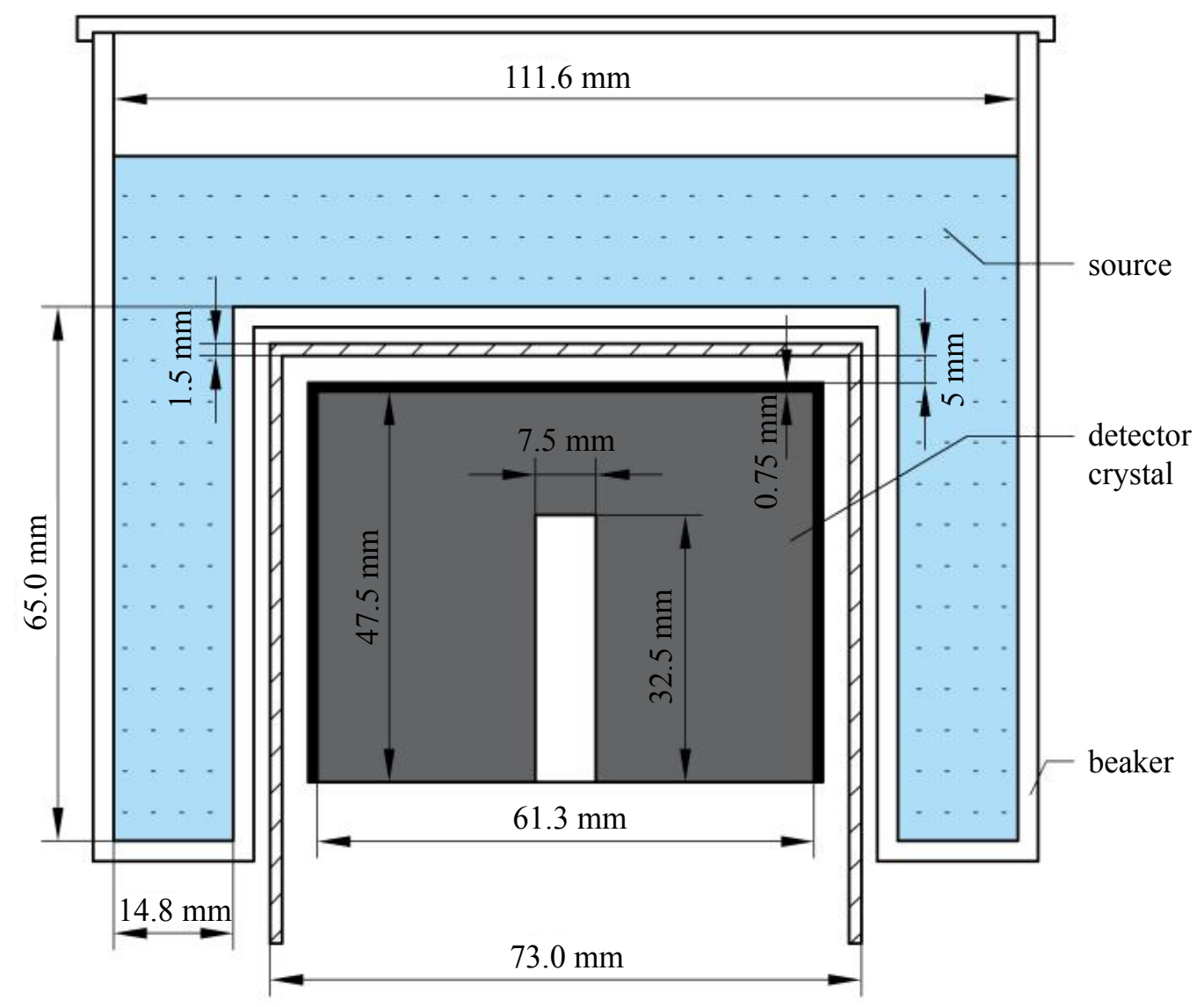

Figure 2: Schematic of the detector with Marinelli beaker source. 
Table 1: Single line and multi gamma ray nuclides with emission probability.

\begin{tabular}{|c|c|c|}
\hline Nuclide & Energy (keV) & $\mathrm{P}(\%)$ \\
\hline${ }^{241} \mathrm{Am}$ & 59.50 & \\
\hline${ }^{109} \mathrm{Cd}$ & 88.02 & \\
\hline${ }^{65} \mathrm{Zn}$ & 111.50 & \\
\hline${ }^{57} \mathrm{Co}$ & 122.06 & \\
\hline${ }^{141} \mathrm{Ce}$ & 145.44 & \\
\hline${ }^{139} \mathrm{Ce}$ & 165.85 & \\
\hline${ }^{51} \mathrm{Cr}$ & 320.08 & \\
\hline${ }^{113} \mathrm{Sn}$ & 391.69 & \\
\hline${ }^{137} \mathrm{Cs}$ & 661.66 & \\
\hline${ }^{54} \mathrm{Mn}$ & 834.84 & \\
\hline \multirow[t]{2}{*}{${ }^{88} \mathrm{Y}$} & 898.02 & 93.70 \\
\hline & 1836.01 & 99.35 \\
\hline \multirow[t]{2}{*}{${ }^{60} \mathrm{Co}$} & 1173.24 & 99.90 \\
\hline & 1332.50 & 99.98 \\
\hline \multirow[t]{4}{*}{${ }^{133} \mathrm{Ba}$} & 276.39 & 7.164 \\
\hline & 160.61 & 0.645 \\
\hline & 302.85 & 18.3334 .06 \\
\hline & 80.99 & \\
\hline \multirow[t]{6}{*}{${ }^{152} \mathrm{Eu}$} & 778. 9 & 13.0626 .78 \\
\hline & 344.3 & 2.79 \\
\hline & 444.0 & 10.12 \\
\hline & 1085.9 & 14.5 \\
\hline & 964.0 & 28.81 \\
\hline & 121.8 & \\
\hline
\end{tabular}

beaker source whose dimension is shown in Figure 2 with volumes V5 $(450 \mathrm{~mL})$, V6 $(600 \mathrm{~mL}), \mathrm{V} 7(800 \mathrm{~mL})$ and $\mathrm{V} 8(1000 \mathrm{~mL})$ was also used in the simulation. The cylinder beaker source was placed at a distance of $6.5 \mathrm{~mm}$ while the Marinelli beaker was placed in contact with the detector end-cap window. The radionuclides contained in the source solution with the $\gamma$-ray emission probability (P) are listed in Table 1. These nuclides were placed within the cylinder volumes at positions $\left(h_{1}=1.6 \mathrm{~mm}, h_{2}=2.6 \mathrm{~mm}\right.$ and $h_{3}=$ $3.6 \mathrm{~mm})$ and $\rho_{\mathrm{i}}(5.32 \mathrm{~mm}, 16.5 \mathrm{~mm}, 25.5 \mathrm{~mm}, 34.5$ $\mathrm{mm}$ and $42.5 \mathrm{~mm}$ ) and Marinelli beaker volumes at $\left(\mathrm{h}_{1}=30 \mathrm{~mm}, \mathrm{~h}_{2}=60 \mathrm{~mm}\right.$ and $\left.\mathrm{h}_{3}=90 \mathrm{~mm}\right)$ and $(22$ $\mathrm{mm}, 44 \mathrm{~mm}, 66 \mathrm{~mm}, 88 \mathrm{~mm}$ and $110 \mathrm{~mm}$ ).

\section{Results and Discussion}

In order to simulate the CSF, the total efficiency is always required with the full energy peak efficiency. The simulated full energy peak and total efficiency curves for cylindrical and Marinelli beaker sources with different volumes are shown in Figure 3 and Figure 4. The figures show that the full energy peak and total efficiency increases for the various volumes with the photon energy around 122.06 $\mathrm{keV}$ where the maximum values for the full energy peak and total efficiency were obtained. The full energy peak and total efficiency are close to each other at the low energy range because the absorption of the $\gamma$-rays in a single photoelectric interaction is predominated only for energies below about $145.44 \mathrm{keV}$ as shown in figures. At high photon energy, the full energy peak efficiency drops off faster than the total efficiency because of the probability of Compton scattering followed by photoelectric absorption of the scattered photon is dominant than the absorption of the full photon energy in a single photoelectric event. As shown in figures the multiple scattering is the dominant contributor to the total efficiency over all but the lowest range of $\gamma$-ray energies. The total efficiency drops off slowly with the increased photon energy due to the less probability of scattered photon in the crystal active volume.

The 15-point integration of efficiency $(\langle J\rangle)$ values obtained with our simulation approach is simple and precise to be used to calculate the CSF. The $\langle\mathrm{J}\rangle$ values of the nuclides ${ }^{60} \mathrm{Co}$ and ${ }^{88} \mathrm{Y}$ for the various source volumes are listed in Table 2 . The <J> values for each source volumes are smaller at low energies and significantly increase at high energy range as shown in Table 2 . The computed $\langle\mathrm{J}\rangle$ value depends on the source volumes. In volumes (50$300 \mathrm{ml})$ and $(450-1000 \mathrm{ml})$, the $<J>$ values decrease with the increase of source volumes for each photon energy. For Marinelli beaker source the $\langle\mathrm{J}\rangle$ value is greater because of the close contact and the small distance of the source inside in the Marinelli beaker to the detector is shown in Table 2.

The CSF values were simulated for cylindrical and Marinelli beaker sources filled with aqueous solution of density $1 \mathrm{~g} / \mathrm{cm}^{3}$. The values of the simulated coincidence summing correction factor $\left(\mathrm{CSF}_{\text {simu }}\right)$ obtained from Eq. 16 and Eq. 19 for $\left({ }^{60} \mathrm{Co}\right.$ and ${ }^{88} \mathrm{Y}$ ) are shown in Table 3 and Table 4 . The $\mathrm{CSF}_{\text {simu }}$ is independent of the detector count rate but it is strongly dependent on the full energy peak and total efficiency. The $\mathrm{CSF}_{\text {simu }}$ values were 


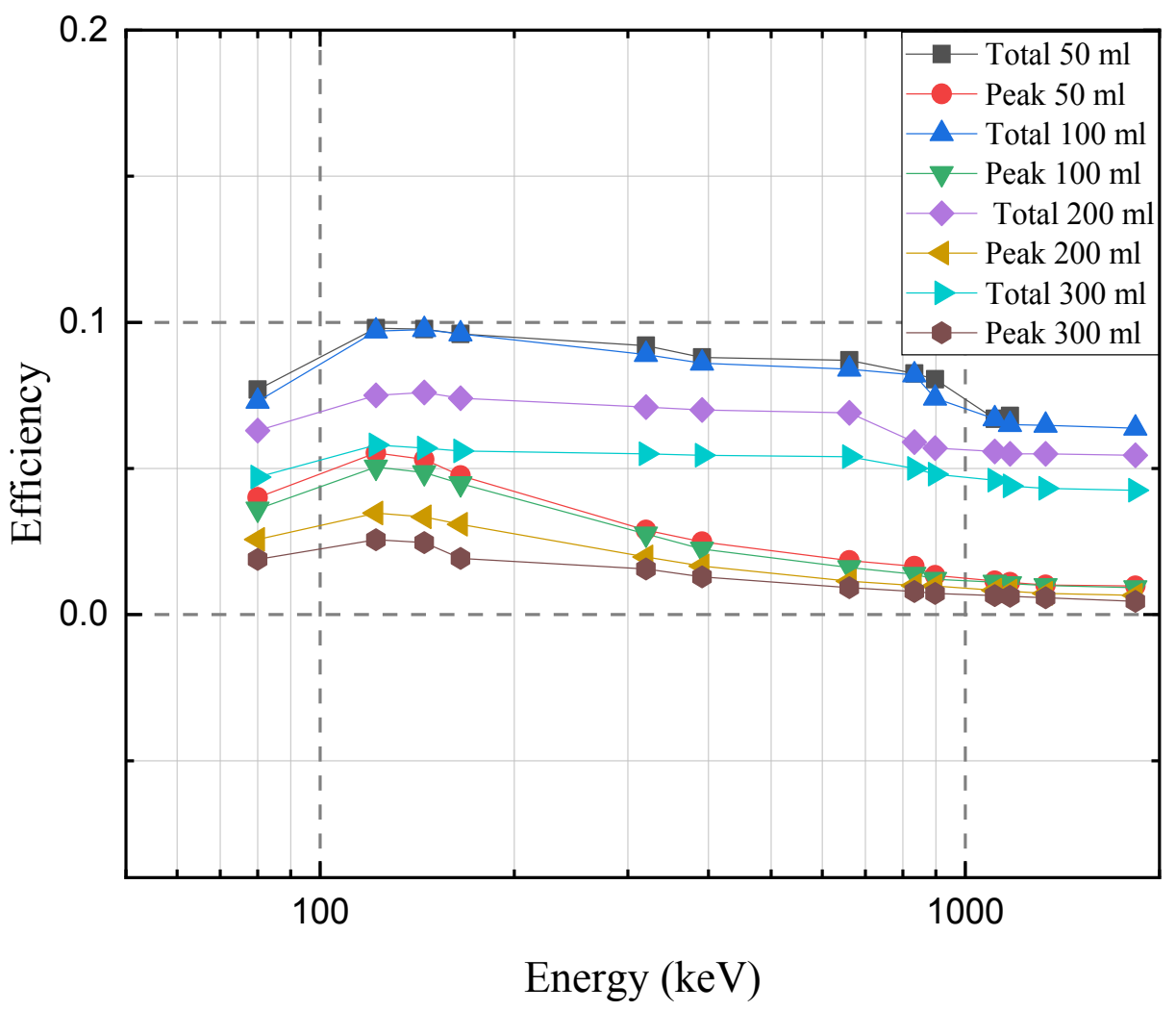

Figure 3: Simulated peak and total efficiencies for cylindrical beaker source.

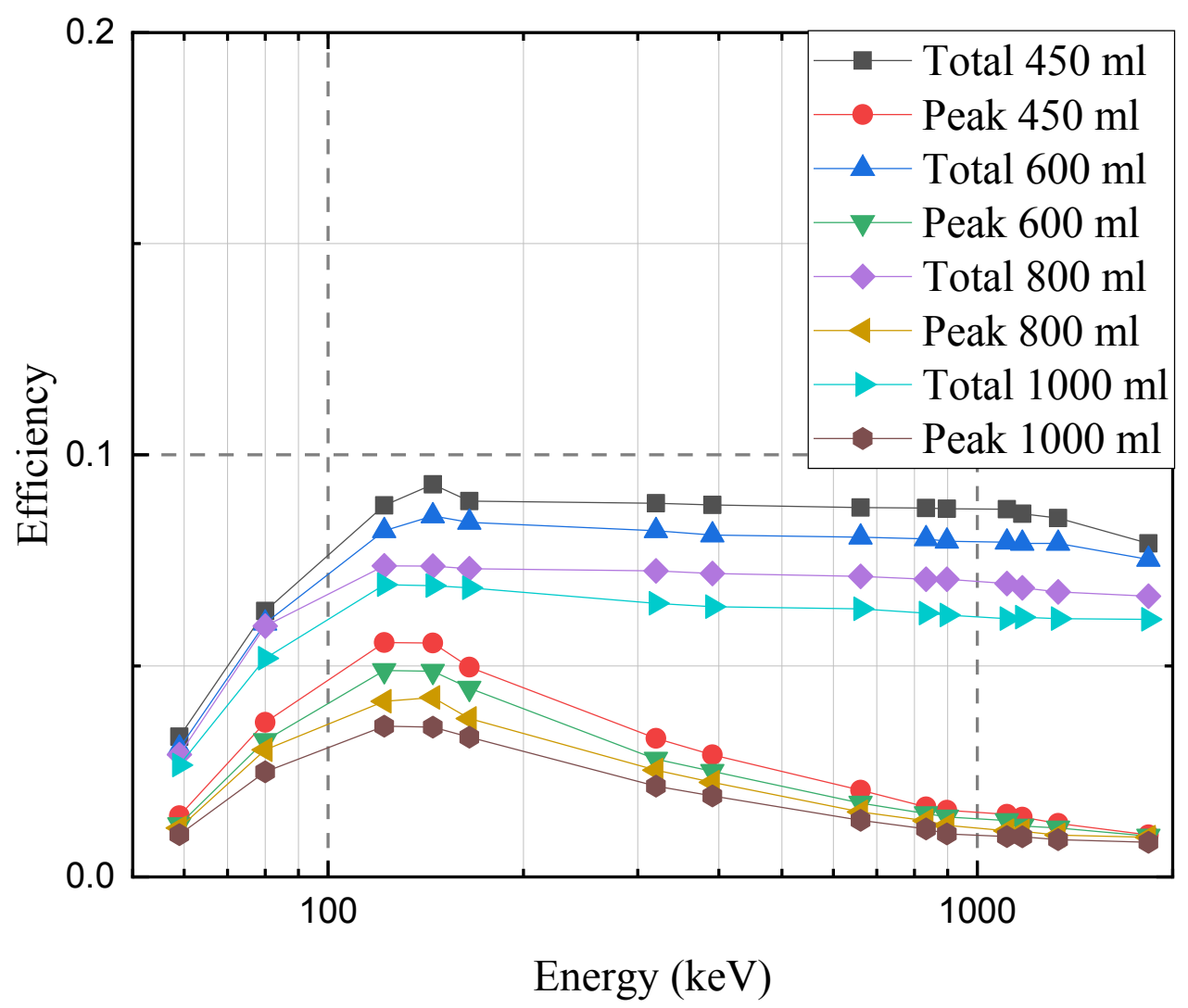

Figure 4: Simulated peak and total efficiencies for Marinelli beaker source. 
Table 2: Computed 15-point integration of efficiency values for cylindrical and Marinelli beaker sources.

\begin{tabular}{|l|l|l|l|l|l|}
\hline Energy (keV) & 15-point integration of efficiencies & Volume (ml) & Volume (ml) & \\
\hline 1173.24 & $\left\langle\mathrm{~J}_{1}\right\rangle$ & 50 & 0.076 & 450 & 0.120 \\
1332.50 & $\left\langle\mathrm{~J}_{1}\right\rangle$ & & 0.078 & & 0.133 \\
898.02 & $\left\langle\mathrm{~J}_{1}\right\rangle$ & & 0.043 & 0.091 \\
1836.01 & $\left\langle\mathrm{~J}_{2}\right\rangle$ & 0.099 & & 0.153 \\
\hline 1173.24 & $\left\langle\mathrm{~J}_{1}\right\rangle$ & 100 & 0.074 & 600 & 0.118 \\
1332.50 & $\left\langle\mathrm{~J}_{2}\right\rangle$ & & 0.075 & & 0.129 \\
898.02 & $\left\langle\mathrm{~J}_{1}\right\rangle$ & & 0.049 & & 0.099 \\
1836.01 & $\left\langle\mathrm{~J}_{2}\right\rangle$ & 0.108 & & 0.169 \\
\hline 1173.24 & $\left\langle\mathrm{~J}_{1}\right\rangle$ & 200 & 0.073 & 800 & 0.114 \\
1332.50 & $\left\langle\mathrm{~J}_{2}\right\rangle$ & & 0.075 & & 0.125 \\
898.02 & $\left\langle\mathrm{~J}_{1}\right\rangle$ & & 0.058 & & 0.104 \\
1836.01 & $\left\langle\mathrm{~J}_{2}\right\rangle$ & & 0.120 & & 0.176 \\
\hline 1173.24 & $\left\langle\mathrm{~J}_{1}\right\rangle$ & 300 & 0.072 & 1000 & 0.111 \\
1332.50 & $\left\langle\mathrm{~J}_{2}\right\rangle$ & & 0.074 & & 0.109 \\
898.02 & $\left\langle\mathrm{~J}_{1}\right\rangle$ & & 0.062 & & 0.107 \\
1836.01 & $\left\langle\mathrm{~J}_{2}\right\rangle$ & & 0.128 & & 0.188 \\
\hline
\end{tabular}

Table 3: Comparison between experimental and simulated coincidence summing correction factors for the cylindrical source.

\begin{tabular}{|c|c|c|c|c|c|}
\hline Volume (ml) & Energy (keV) & CSF $_{\text {Exp }}$ & $\mathbf{C S F}_{\mathrm{Cal}}$ & CSF $_{\text {simu }}$ & RD (\%) \\
\hline \multirow[t]{4}{*}{50} & 1173.24 & 0.917 & 0.923 & 0.924 & -0.7 \\
\hline & 1332.50 & 0.916 & 0.921 & 0.922 & -0.6 \\
\hline & 898.02 & & & 0.957 & \\
\hline & 1836.01 & & & 0.901 & \\
\hline \multirow[t]{4}{*}{100} & 1173.24 & 0.921 & 0.927 & 0.926 & -0.5 \\
\hline & 1332.50 & 0.905 & 0.925 & 0.925 & -2.1 \\
\hline & 898.02 & & & 0.951 & \\
\hline & 1836.01 & & & 0.892 & \\
\hline \multirow[t]{4}{*}{200} & 1173.24 & 0.923 & 0.935 & 0.927 & -0.4 \\
\hline & 1332.50 & 0.911 & 0.934 & 0.925 & -1.5 \\
\hline & 898.02 & & & 0.942 & \\
\hline & 1836.01 & & & 0.880 & \\
\hline \multirow[t]{4}{*}{300} & 1173.24 & 0.922 & 0.938 & 0.928 & -0.6 \\
\hline & 1332.50 & 0.905 & 0.937 & 0.926 & -2.2 \\
\hline & 898.02 & & & 0.938 & \\
\hline & 1836.01 & & & 0.872 & \\
\hline
\end{tabular}

compared with the experimental and calculated results and obtained good agreement with the relative deviation equal to $2 \%$. For each multi $\gamma$-ray nuclide, the $\mathrm{CSF}_{\text {simu }}$ value is somewhat greater at low photon energy because of the greater $\langle J\rangle$ value at high photon energy, which means that there is an 
Table 4: Comparison between experimental and simulated coincidence summing correction factors for Marinelli beaker source.

\begin{tabular}{|l|l|l|l|l|l|}
\hline Volume (ml) & Energy (keV) & CSF $_{\text {Exp }}$ & CSF $_{\text {Cal }}$ & CSF $_{\text {simu }}$ & RD (\%) \\
\hline 450 & 1173.24 & 0.875 & 0.901 & 0.880 & -0.5 \\
\hline & 1332.50 & 0.858 & 0.900 & 0.867 & -0.1 \\
\hline & 898.02 & & & 0.909 & \\
\hline & 1836.01 & & & 0.847 & \\
\hline & 1173.24 & 0.874 & 0.906 & 0.882 & -0.9 \\
\hline & 1332.50 & 0.867 & 0.905 & 0.871 & -0.4 \\
\hline & 898.02 & & & 0.901 & \\
\hline & 1836.01 & & & 0.831 & \\
\hline & 1173.24 & 0.872 & 0.909 & 0.886 & -1.6 \\
\hline & 1332.50 & 0.868 & 0.908 & 0.875 & -0.8 \\
\hline & 898.02 & & & 0.896 & \\
\hline & 1836.01 & & & 0.824 & -0.5 \\
\hline & 1173.24 & 0.884 & 0.911 & 0.889 & -1.5 \\
\hline & 1332.50 & 0.877 & 0.910 & 0.891 & \\
\hline & 898.02 & & & 0.893 & \\
\hline & 1836.01 & & & 0.812 & \\
\hline
\end{tabular}

Table 5: Comparison of the simulated coincidence summing correction factors for different densities.

\begin{tabular}{|l|l|l|l|l|}
\hline Volume (ml) & Energy $(\mathbf{k e V})$ & CSF $_{\text {simu }}$ & Density & $\boldsymbol{\rho}\left(\mathbf{g} / \mathbf{c m}^{\mathbf{3}} \mathbf{~}\right.$ \\
\hline 50 & 1173.24 & 0.920 & 0.924 & 0.945 \\
\hline \multirow{2}{*}{100} & 1332.50 & 0.916 & 0.922 & 0.945 \\
\hline \multirow{2}{*}{200} & 1173.24 & 0.922 & 0.926 & 0.950 \\
\hline & 1332.50 & 0.917 & 0.925 & 0.947 \\
\hline 300 & 1173.24 & 0.923 & 0.927 & 0.962 \\
\hline \multirow{2}{*}{450} & 1332.50 & 0.917 & 0.925 & 0.962 \\
\hline \multirow{2}{*}{600} & 1173.24 & 0.924 & 0.928 & 0.971 \\
\hline 800 & 1332.50 & 0.920 & 0.926 & 0.970 \\
\hline 1000 & 1173.24 & 0.859 & 0.880 & 0.931 \\
\hline & 1332.50 & 0.832 & 0.867 & 0.929 \\
\hline & 1173.24 & 0.828 & 0.882 & 0.937 \\
\hline & 1332.50 & 0.822 & 0.871 & 0.936 \\
\hline & 1173.24 & 0.810 & 0.886 & 0.948 \\
\hline & 1332.50 & 0.801 & 0.875 & 0.946 \\
\hline & 1173.24 & 0.781 & 0.889 & 0.957 \\
\hline & 1332.50 & 0.772 & 0.891 & 0.956 \\
\hline
\end{tabular}

inverse relationship between $<\mathrm{J}>$ and $\mathrm{CSF}_{\text {simu }}$ values. tively. The comparison of the $\mathrm{CSF}_{\text {simu }}$ values for cyTo observe the sample density effect on the CSFvalue, the simulation was performed for ethanol, water and sea sand sample (major component $\mathrm{SiO}_{2}$ ) with densities $\left(0.7,1\right.$ and $\left.2.5 \mathrm{~g} / \mathrm{cm}^{3}\right)$ respeclindrical and Marinelli beaker sources with different sample density are shown in Table 5 . When the density of sample increases the $\mathrm{CSF}_{\text {simu }}$ value increases because the minimum number of $\gamma$-rays 
Table 6: Comparison of experimental and simulated coincidence summing correction factors of 133Ba for cylindrical source.

\begin{tabular}{|l|l|l|l|l|l|l|}
\hline Volumes (ml) & Energy (keV) & CSF $_{\text {Exp }}$ & CSF $_{\text {Cal }}$ & $\left\langle\mathrm{J}_{1}\right\rangle\left\langle\mathrm{J}_{2}\right\rangle$ & CSF $_{\text {Simu }}$ & RD (\%) \\
\hline 50 & 276.39 & 0.915 & 0.924 & 0.062 & 0.938 & -2.5 \\
\hline \multirow{2}{*}{100} & 302.85 & 0.937 & 0.959 & 0.033 & 0.967 & -3.2 \\
\hline \multirow{2}{*}{200} & 276.39 & 0.934 & 0.925 & 0.069 & 0.931 & 0.3 \\
\hline \multirow{2}{*}{300} & 302.85 & 0.944 & 0.959 & 0.042 & 0.958 & -1.4 \\
\hline & 276.39 & 0.929 & 0.934 & 0.071 & 0.929 & 0.0 \\
\hline & 302.85 & 0.952 & 0.965 & 0.050 & 0.950 & 0.2 \\
\hline & 276.39 & 0.932 & 0.937 & 0.040 & 0.960 & -3.0 \\
\hline & 302.85 & 0.958 & 0.967 & 0.030 & 0.970 & -1.2 \\
\hline
\end{tabular}

Table 7: Comparison of experimental and simulated coincidence summing correction factors of ${ }^{133} \mathrm{Ba}$ for Marinelli beaker source.

\begin{tabular}{|l|l|l|l|l|l|l|}
\hline Volumes (ml) & Energy (keV) & CSF $_{\text {Exp }}$ & CSF $_{\text {Cal }}$ & $\left\langle\mathrm{J}_{1}\right\rangle\left\langle\mathrm{J}_{2}\right\rangle$ & CSF $_{\text {simu }}$ & RD (\%) \\
\hline 450 & 276.39 & 0.927 & 0.918 & 0.086 & 0.914 & 1.4 \\
\hline \multirow{2}{*}{600} & 302.85 & 0.947 & 0.958 & 0.035 & 0.965 & -1.9 \\
\hline \multirow{2}{*}{800} & 276.39 & 0.920 & 0.923 & 0.062 & 0.938 & -1.9 \\
\cline { 2 - 7 } & 302.85 & 0.945 & 0.961 & 0.050 & 0.950 & -0.5 \\
\hline \multirow{2}{*}{1000} & 276.39 & 0.932 & 0.924 & 0.064 & 0.936 & -0.4 \\
\hline & 302.85 & 0.957 & 0.961 & 0.040 & 0.960 & -0.3 \\
\hline & 276.39 & 0.932 & 0.924 & 0.066 & 0.934 & -0.2 \\
\hline & 302.85 & 0.958 & 0.961 & 0.031 & 0.969 & -1.1 \\
\hline
\end{tabular}

scattered in the samples itself at greater density. This analysis shows that the CSF value increased with the self-absorption of the source matrix.

The proposed simulated method was also applied to obtain the CSF values of ${ }^{133} \mathrm{~B}$ and ${ }^{152} \mathrm{Eu}$. The $\mathrm{CSF}_{\text {simu }}$ value for ${ }^{133} \mathrm{~B}(276.39 \mathrm{keV})$ was calculated using Eq. 16 with total efficiency of $160.61 \mathrm{keV}$. Similarly, $\mathrm{CSF}_{\text {simu }}$ value was calculated for $302.85 \mathrm{keV}$ using Eq. 19 with emission probability ratio $\left(\frac{\mathrm{p}_{1}}{\mathrm{p}_{2}}\right)$ of ( $80.99 \mathrm{keV}$ and $302.85 \mathrm{keV}$ ) and total efficiency of $80.99 \mathrm{keV}$. The simulated values were compared with the experimental results for cylindrical and Marinelli beaker sources as shown in Table 6 and Table 7. The simulated results agreed with the experimental values within $2 \%$ for all source volumes, except for the $50 \mathrm{ml}$ and $300 \mathrm{ml}$ where they are up to $3 \%$. In the case of ${ }^{152} \mathrm{Eu}$, Eq.16 was used to calculate the $\mathrm{CSF}_{\text {simu }}$ value for $(778.9 \mathrm{KeV}, 964.0 \mathrm{KeV}$ and $444.0 \mathrm{KeV}$ ) with respect to the total efficiency of $344.3 \mathrm{keV}, 1085.9 \mathrm{keV}$ and $121.8 \mathrm{keV}$. The sim- ulated results were compared with the experimental and calculated CSF values and obtained good agreements with experimental as shown in Table 8 and Table 9.

\section{Conclusions}

A new method was used in GEANT4 to calculate the coincidence summing correction factors from the peak and total efficiencies and obtained accurate results for ${ }^{60} \mathrm{Co},{ }^{88} \mathrm{Y},{ }^{133} \mathrm{Ba}$ and ${ }^{152} \mathrm{Eu}$, the average discrepancies between the experimental and simulated results were less than $1 \%$. The simulation was performed and obtained the coincidence summing correction factors for various source volumes and observed the dependence of correction factors value on different samples densities. An easy technique developed in this study for the calculation of coincidence summing correction factor of complex nuclides. The suggested simulation method avoids the preparation of a great variety of gaseous samples with several isotopes and has added the advantages to improve the detection efficiencies for 
Table 8: Comparison of experimental and simulated coincidence summing correction factors of 152Eu for cylindrical source.

\begin{tabular}{|l|l|l|l|l|l|l|}
\hline Volumes (ml) & Energy (keV) & CSF $_{\text {Exp }}$ & CSF $_{\text {Cal }}$ & $\left\langle\mathrm{J}_{1}\right\rangle$ & CSF $_{\text {Simu }}$ & RD (\%) \\
\hline 50 & 778.9 & 0.903 & 0.906 & 0.083 & 0.917 & -1.5 \\
\hline & 964.0 & 0.920 & 0.933 & 0.065 & 0.935 & -0.2 \\
\hline & 444.0 & 0.902 & 0.884 & 0.086 & 0.914 & -3.0 \\
\hline \multirow{3}{*}{100} & 778.9 & 0.894 & 0.911 & 0.091 & 0.909 & -1.6 \\
\hline & 964.0 & 0.914 & 0.935 & 0.077 & 0.923 & -0.9 \\
\hline \multirow{2}{*}{200} & 444.0 & 0.914 & 0.889 & 0.083 & 0.917 & -0.3 \\
\hline & 778.9 & 0.907 & 0.921 & 0.078 & 0.922 & -1.6 \\
\hline 300 & 964.0 & 0.925 & 0.942 & 0.067 & 0.933 & -0.8 \\
\hline & 444.0 & 0.927 & 0.901 & 0.065 & 0.935 & -0.8 \\
\hline & 778.9 & 0.916 & 0.924 & 0.071 & 0.929 & -1.4 \\
\hline & 964.0 & 0.923 & 0.946 & 0.073 & 0.927 & -0.4 \\
\hline & 444.0 & 0.932 & 0.905 & 0.058 & 0.942 & -1.0 \\
\hline
\end{tabular}

Table 9: Comparison of experimental and simulated coincidence summing correction factors of ${ }^{152} \mathrm{Eu}$ for Marinelli beaker source.

\begin{tabular}{|l|l|l|l|l|l|l|}
\hline Volumes (ml) & Energy (keV) & CSF $_{\text {Exp }}$ & CSF $_{\text {Cal }}$ & $\left\langle\mathrm{J}_{1}\right\rangle$ & CSF $_{\text {Simu }}$ & RD (\%) \\
\hline 450 & 778.9 & 0.870 & 0.887 & 0.111 & 0.889 & -2.0 \\
\hline & 964.0 & 0.894 & 0.922 & 0.922 & 0.912 & -2.0 \\
\cline { 2 - 7 } & 444.0 & 0.888 & 0.859 & 0.095 & 0.905 & -1.9 \\
\hline 600 & 778.9 & 0.967 & 0.893 & 0.030 & 0.970 & -0.3 \\
\hline \multirow{3}{*}{800} & 964.0 & 0.899 & 0.927 & 0.085 & 0.915 & -1.7 \\
\hline & 444.0 & 0.894 & 0.867 & 0.088 & 0.912 & -2.0 \\
\hline \multirow{3}{*}{1000} & 778.9 & 0.870 & 0.896 & 0.110 & 0.890 & -2.2 \\
\hline & 964.0 & 0.895 & 0.929 & 0.090 & 0.910 & -1.6 \\
\hline & 444.0 & 0.894 & 0.870 & 0.095 & 0.905 & -1.2 \\
\hline & 778.9 & 0.875 & 0.897 & 0.108 & 0.892 & -1.9 \\
\hline & 964.0 & 0.904 & 0.930 & 0.087 & 0.913 & -0.9 \\
\hline & 444.0 & 0.908 & 0.972 & 0.083 & 0.917 & -0.9 \\
\hline
\end{tabular}

the measurement of the activity of environmental samples.

\section{Acknowledgments}

This work at Xian Jiaotong University was fully supported by the Chinese government.

\section{References}

1. K Debertin, U Schötzig (1979) Coincidence summing corrections in Ge (Li)-spectrometry at low sourceto-detector distances. Nuclear Instruments and Methods 158: 471-477.
2. MI Abbas (2001) HPGe detector photopeak efficiency calculation including self-absorption and coincidence corrections for Marinelli beaker sources using compact analytical expressions. Applied Radiation and Isotopes 54: 761-768.

3. H Jäderström, W Mueller, V Atrashkevich, A Adekola (2015) True coincidence summing correction and mathematical efficiency modeling of a well detector. Nuclear Instruments and Methods in Physics Research Section A: Accelerators, Spectrometers, Detectors and Associated Equipment 784: 264-268.

4. TK Wang, WY Mar, TH Ying, CH Liao, CL Tseng (1995) 
HPGe detector absolute-peak-efficiency calibration by using the ESOLAN program. Appl Radiat Isot 46: 933-944.

5. MI Abbas (2007) Direct mathematical method for calculating full-energy peak efficiency and coincidence corrections of HPGe detectors for extended sources. Nuclear Instruments and Methods in Physics Research Section B: Beam Interactions with Materials and Atoms 256: 554-557.

6. E Tomarchio, S Rizzo (2011) Coincidence-summing correction equations in gamma-ray spectrometry with p-type HPGedetectors. Radiation Physics and Chemistry 80: 318-323.

7. M Lee, TS Park, JK Woo (2008) Coincidence summing effects in gamma-ray spectrometry using a Marinelli beaker. Appl Radiat Isot 66: 799-803.

8. MS Badawi, SI Jovanovic, AA Thabet, AM El-Khatib, AD Dlabac, et al. (2017) Calibration of $4 \pi \mathrm{Nal}(\mathrm{TI})$ detectors with coincidence summing correction using new numerical procedure and ANGLE4 software. AIP Advances 7: 035005.

9. AM El-Khatib, BA Salem, MS Badawi, MM Gouda, AA Thabet, et al. (2017) Full-Energy peak efficiency of an $\mathrm{Nal}(\mathrm{TI})$ detector with coincidence summing correction showing the effect of the source-to-detector distance, Chinese Journal of Physics 55: 478-489.

10.Z Wang, B Kahn, JD Valentine (2002) Efficiency calculation and coincidence summing correction for germanium detectors by Monte Carlo simulation. IEEE Transactions on Nuclear Science 49: 1925-1931.

11.T Vidmar, A Likar (2005) Calculation of total efficiencies of extended samples for HPGe detectors, Nuclear Instruments and Methods in Physics
Research Section A: Accelerators, Spectrometers, Detectors and Associated Equipment 555: 251-254.

12.AM Ababneh, MM Eyadeh (2015) Coincidence summing corrections in HPGe gamma-ray spectrometry for Marinelli-beakers geometry using peak to total $(P / T)$ calibration. Journal of Radiation Research and Applied Sciences 8: 323-327.

13.T Vidmar, M Korun, B Vodenik (2007) A method for calculation of true coincidence summing correction factors for extended sources. Applied radiation and isotopes 65: 243-246.

14.D Arnold, O Sima (2004) Application of GESPECOR software for the calculation of coincidence summing effects in special cases. Applied radiation and isotopes 60: 167-172.

15.M Blaauw, SJ Gelsema (2003) Cascade summing in gamma-ray spectrometry in marinelli-beaker geometries: the third efficiency curve. Nuclear Instruments and Methods in Physics Research Section A: Accelerators, Spectrometers, Detectors and Associated Equipment 505: 311-315.

16.S Hurtado, M Garcia-León, R Garcia-Tenorio (2004) GEANT4 code for simulation of a germanium gamma-ray detector and its application to efficiency calibration. Nucl Instr Meth Phys Res A 518: 764-774.

17.B Quintana, C Montes (2014) Summing-coincidence corrections with Geant4 in routine measurements by $\gamma$ spectrometry of environmental samples. Applied Radiation and Isotopes 87: 390-393.

18.W Khan, Q Zhang, C He, M Saleh, (2018) Monte Carlo simulation of the full energy peak efficiency of an HPGe detector. Applied Radiation and Isotopes 131: 67-70. 